\title{
DOT tomography of the solar atmosphere
}

\section{Magnetic patches in internetwork areas}

\author{
A. G. de Wijn ${ }^{1}$, R. J. Rutten ${ }^{1,2}$, E. M. W. P. Haverkamp ${ }^{1}$, and P. Sütterlin ${ }^{1}$
}

1 Sterrekundig Instituut, Utrecht University, Postbus 80 000, 3508 TA Utrecht, The Netherlands
e-mail: [A.G.deWijn; R. J . Rutten] @astro.uu.nl
2 Institute of Theoretical Astrophysics, Oslo University, PO Box 1029 Blindern, 0315 Oslo, Norway

Received 5 May 2005 / Accepted 23 June 2005

ABSTRACT

We use G-band and Ca II H image sequences from the Dutch Open Telescope (DOT) to study magnetic elements that appear as bright points in internetwork parts of the quiet solar photosphere and chromosphere. We find that many of these bright points appear recurrently with varying intensity and horizontal motion within longer-lived magnetic patches. We develop an algorithm for detection of the patches and find that all patches identified last much longer than the granulation. The patches outline cell patterns on mesogranular scales, indicating that magnetic flux tubes are advected by granular flows to mesogranular boundaries. Statistical analysis of the emergence and disappearance of the patches points to an average patch lifetime as long as $530 \pm 50 \mathrm{~min}$ (about nine hours), which suggests that the magnetic elements constituting strong internetwork fields are not generated by a local turbulent dynamo.

Key words. Sun: magnetic fields - Sun: granulation - Sun: photosphere - Sun: chromosphere

\section{Introduction}

In this paper, we address the appearance and lifetime of magnetic elements that intermittently show up as bright points in the internetwork areas of the quiet sun. The context is the nature of quiet-sun magnetism, its dynamical coupling to transitionregion and coronal fields, and the existence of a local turbulent dynamo.

The more familiar network bright points are similar. They constitute the magnetic network which partially outlines the boundaries of supergranular cells, and have long been recognized to represent strong-field magnetic elements, which are traditionally modeled as flux tubes. They were first observed as magnetic knots (Beckers \& Schröter 1968) and as "filigree" (Dunn \& Zirker 1973) resolved into strings of adjacent bright points by Mehltretter (1974). Prompted by Muller (1977), Wilson (1981) showed that faculae, filigree, and bright points in wide-band $\mathrm{Ca}$ II $\mathrm{H}$ filtergrams are manifestations of the same phenomenon. Muller (1983) subsequently introduced the name "network bright point" (NBP) and initiated an extensive literature observing them as G-band bright points (Muller \& Roudier 1984). In particular the G-band studies with the former and the present Swedish solar telescope on La Palma (Berger et al. 1995, 1998a,b, 2004; Berger \& Title 1996, 2001; Löfdahl et al. 1998; van Ballegooijen et al. 1998; Wiehr et al. 2004; Rouppe van der Voort et al. 2005) established that NBPs are brightness manifestations of the small, strongfield magnetic elements that make up the magnetic network
(Chapman \& Sheeley 1968; Livingston \& Harvey 1969; Howard \& Stenflo 1972; Frazier \& Stenflo 1972; Stenflo 1973). These have been modeled as magnetostatic flux tubes since the pioneering work of Spruit $(1976,1977)$ inspired by Zwaan (1967). Their hot-wall explanation of the photospheric brightness enhancement (Spruit 1976; Spruit \& Zwaan 1981) was recently verified by the MHD simulations of Keller et al. (2004) and Carlsson et al. (2004), which crown a long effort in flux tube modeling (e.g., Knölker \& Schüssler 1988; Keller et al. 1990; Solanki \& Brigljevic 1992; Grossmann-Doerth et al. 1994, 1998; Steiner et al. 1998; Steiner 2005). NBPs serve as tracers of strong-field flux tubes, especially in the Fraunhofer $\mathrm{G}$ band $(\mathrm{CH}$ lines around $430.5 \mathrm{~nm})$ and the $\mathrm{CN}$ band at $388 \mathrm{~nm}$ (cf. Rutten et al. 2001), but with the caveat that not all magnetic features produce observable bright points (Berger \& Title 2001).

The nature of solar magnetism in the quiet-sun internetwork areas, i.e., the supergranular cell interiors bordered incompletely by filigree chains and NBPs that form the network, is less well established, but is presently under intense scrutiny (e.g., Domínguez Cerdeña et al. 2003; Sánchez Almeida et al. 2003; Socas-Navarro et al. 2004; Lites \& Socas-Navarro 2004; Socas-Navarro \& Lites 2004; Trujillo Bueno et al. 2004; Manso Sainz et al. 2004). A full spectrum of field strengths seems to be ubiquitously present in the internetwork at small spatial scales, with the stronger elements residing in intergranular lanes. When strong enough, such internetwork elements are 
sufficiently evacuated in the low photosphere to appear as internetwork bright points (IBPs) that are quite similar to NBPs, but more isolated. Their existence was already noted by Muller (1983), but only recently a more detailed study of photospheric IBPs was presented by Sánchez Almeida et al. (2004). They report a density of 0.3 IBP per $\mathrm{Mm}^{2}$ and lifetimes of a few minutes, shorter than the average NBP lifetime of $9.3 \mathrm{~min}$ measured by Berger et al. (1998b).

It is advantageous to combine photospheric imaging in the $\mathrm{G}$ band with simultaneous co-spatial imaging in the core of the Ca II $\mathrm{H}$ line to study IBP appearance, patterning, and lifetimes. In Ca II H \& K images sampling the low chromosphere, both NBPs and IBPs show up with a larger brightness enhancement over the surrounding area than they do in G-band or continuum images sampling the photosphere (cf. Fig. 2 of Lites et al. 1999). Ca II H imaging therefore provides a better diagnostic to detect isolated IBPs, for which contrast is more important than sharpness. The latter is lower for $\mathrm{Ca}$ II $\mathrm{H} \& \mathrm{~K}$ images due to strong scattering, and possibly also due to increasing flux tube width with height. G-band IBPs vanish sooner than their Ca II counterparts when they diminish in brightness. G-band imaging at the highest resolution is needed to faithfully render the intricate brightness structure of individual magnetic elements (Berger et al. 2004), but synchronous Ca II imaging provides better location detection especially for isolated ones, as IBPs often are.

Intermittent IBPs observed in Ca II H \& K were called "persistent flashers" by Brandt et al. (1992, 1994), who described a particular $\mathrm{Ca}$ II $\mathrm{K}_{2 V}$ grain which appeared and disappeared during multiple hours while migrating from the center to the boundary of a cell. The grain followed a flow path determined from independent granulation tracking as if it were a cork floating on the solar surface. These flashers figured in the extensive debate whether all briefly-appearing bright grains in $\mathrm{Ca}$ II $\mathrm{H} \& \mathrm{~K}$ image sequences, in particular at the $\mathrm{H}_{2 V}$ and $\mathrm{K}_{2 V}$ off-center wavelengths, represent non-magnetic acoustic shocks or magnetism-constrained phenomena (e.g., Sivaraman \& Livingston 1982; Rutten \& Uitenbroek 1991; von Uexkuell \& Kneer 1995; Carlsson \& Stein 1997; Nindos \& Zirin 1998; Worden et al. 1999; Lites et al. 1999; Sivaraman et al. 2000). The conclusion is that the acoustic internetwork grains appear only a few times with a modulation of roughly three minutes, whereas the strongest internetwork magnetic elements stand out by their "persistent flasher" character: they possess an apparent location memory which significantly exceeds a few three-minute cycles. Many such longer-lived (while modulated) internetwork brightness features were noted in ultraviolet image sequences from TRACE by Krijger et al. (2001), who displayed one in their Fig. 7. A higher-resolution example from the Dutch Open Telescope (DOT) is shown in Fig. 2 of Rutten et al. (2004a).

In this paper, we study IBPs in synchronous G-band and $\mathrm{Ca}$ II $\mathrm{H}$ image sequences with high resolution and fast cadence, as a sequel to the remark by Rutten et al. (2004a) that the IBP identified as magnetic flasher there persisted over the full sequence duration of $54 \mathrm{~min}$. The main issue is whether briefly appearing IPBs systematically portray longer-lived flux concentrations that vary temporally in their morphology and intensity. Our Ca II H imaging and statistical analysis below indeed suggest that this is the case. This result is important in the context of field generation by turbulent dynamos (Cattaneo 1999; Emonet \& Cattaneo 2001; Cattaneo et al. 2003), of the field topology surrounding network at chromospheric heights (cf. Schrijver \& Title 2003), and of the internetwork contribution to coronal heating by wave dissipation (e.g., Heyvaerts \& Priest 1983) and reconnection (e.g., Parker 1988) imposed by photospheric foot point motions and topology evolution.

\section{Observations, data reduction, and patch identification}

We use a double image sequence of a quiet area at the disk center recorded by the DOT from 8:40 to 9:39 UT on June 16, 2003. The sequences consist of speckle-reconstructed images taken at a 20-s cadence in the Fraunhofer $G$ band with a 10 - $\AA$ filter centered at $4305 \AA$, and synchronous, specklereconstructed images taken in the Ca II H line (3968 $\mathrm{A})$ with a narrow-band filter ( $F W H M 1.3 \AA$ ) at line center. A sample pair of G-band and Ca II H images is shown in Fig. 1. Details on the telescope, its tomographic multi-wavelength imaging and image acquisition, and the speckle reconstruction and standard reduction procedures are given in Rutten et al. (2004b).

The images were carefully aligned and destretched using Fourier correlation. After clipping to the common field of view $\left(68 \times 63 \operatorname{arcsec}^{2}\right)$, the resulting sequences consist of 178 speckle-reconstructed image pairs of $962 \times 894$ square pixels of $0.071^{\prime \prime}$ size. Each sequence was cone-filtered in Fourier space to remove features that travel with apparent horizontal speed exceeding the $7 \mathrm{~km} \mathrm{~s}^{-1}$ sound speed. The image sequences may be downloaded as movies from the DOT database $^{1}$, together with synchronous blue and red continuum sequences not used in this analysis.

In order to visually search for recurrent IBPs, we employed an interactive three-dimensional "cube slicer", which dissects both data cubes simultaneously in $x-y, x-t$, and $y-t$ slices with continuous $(x, y, t)$ selection controlled by mouse movement. For example, IBPs show up in the $x-t$ slice only when not drifting in $y$, but a slight wiggling of the $y$ coordinate then helps to track the three-dimensional "world-line" of the feature through both data cubes. We so found many IBPs that are intermittently present while migrating slightly in $x$ and/or $y$. They are easiest to detect in the internetwork parts of the $\mathrm{Ca}$ II $\mathrm{H}$ sequence, but are accompanied, at least for part of the time, by far sharper cospatial G-band IBPs in the underlying photosphere. Often, we find groups of recurrent IBPs that appear associated through a shared magnetic structure providing a longer-term spatial location memory. We call such linked IBP groups "magnetic patches". They frequently consist of multiple strings of IBPs that split and merge.

Figure 2 displays an illustrative example. The panel layout mimics cube slicing in the form of a sequence of small successive $x-y$ image cutouts (top row) and a sequence of $x-t$ slice cutouts stepping progressively in their $y$ sampling location (middle row). The rightmost column of panels are averages

\footnotetext{
${ }^{1}$ http://dot.astro.uu.nl/
} 

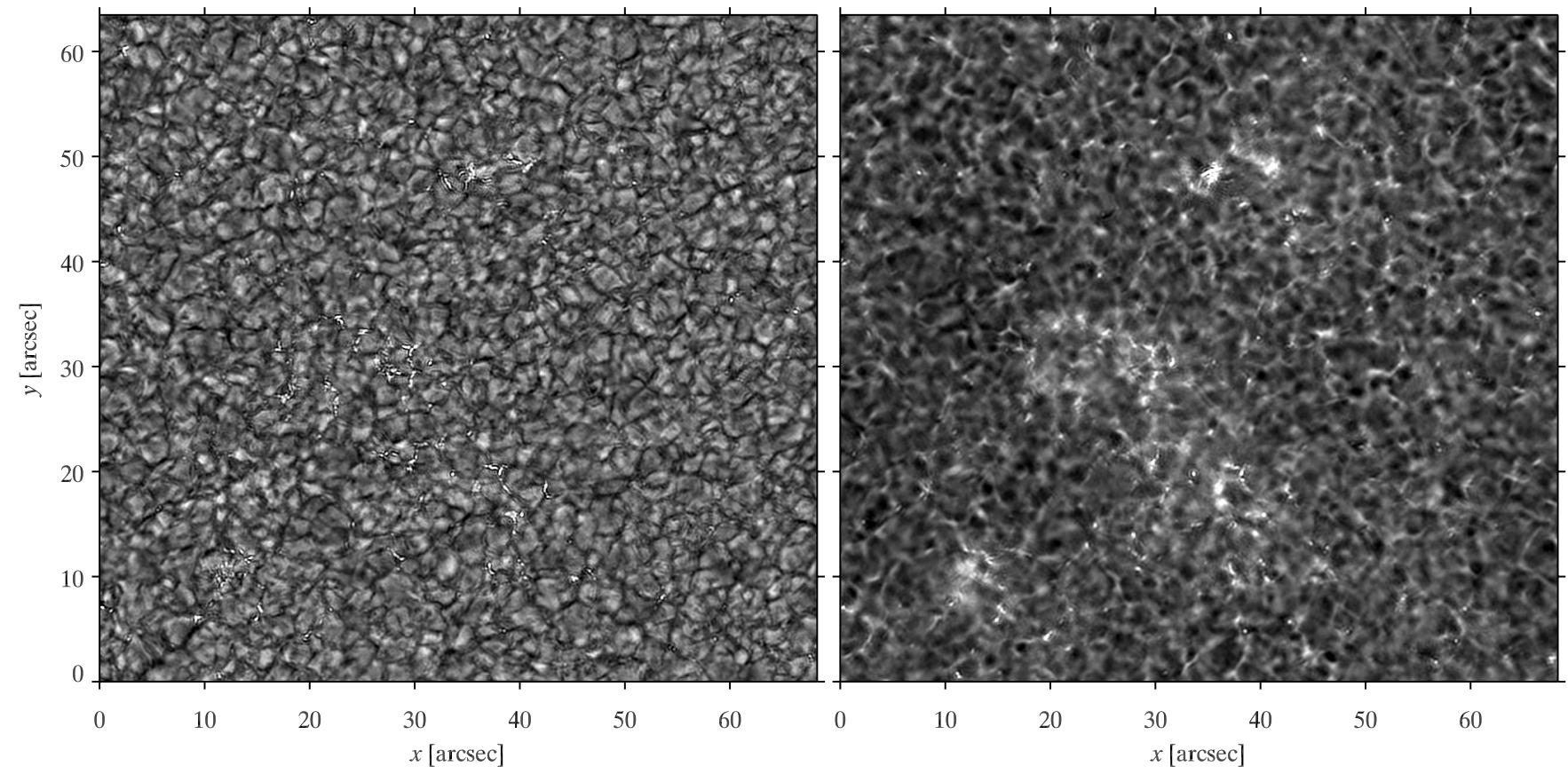

Fig. 1. A sample image pair from the Dutch Open Telescope (DOT). Left: G-band image. Right: co-temporal and co-spatial Ca II H image, clipped to improve contrast. The network is readily identified in the Ca II H image as regions with enhanced brightness. In the G-band image these areas contain many tiny bright points, often arranged in strings (filigree) within intergranular lanes. These are NBPs. Close inspection shows the presence of isolated IBPs in the internetwork regions.
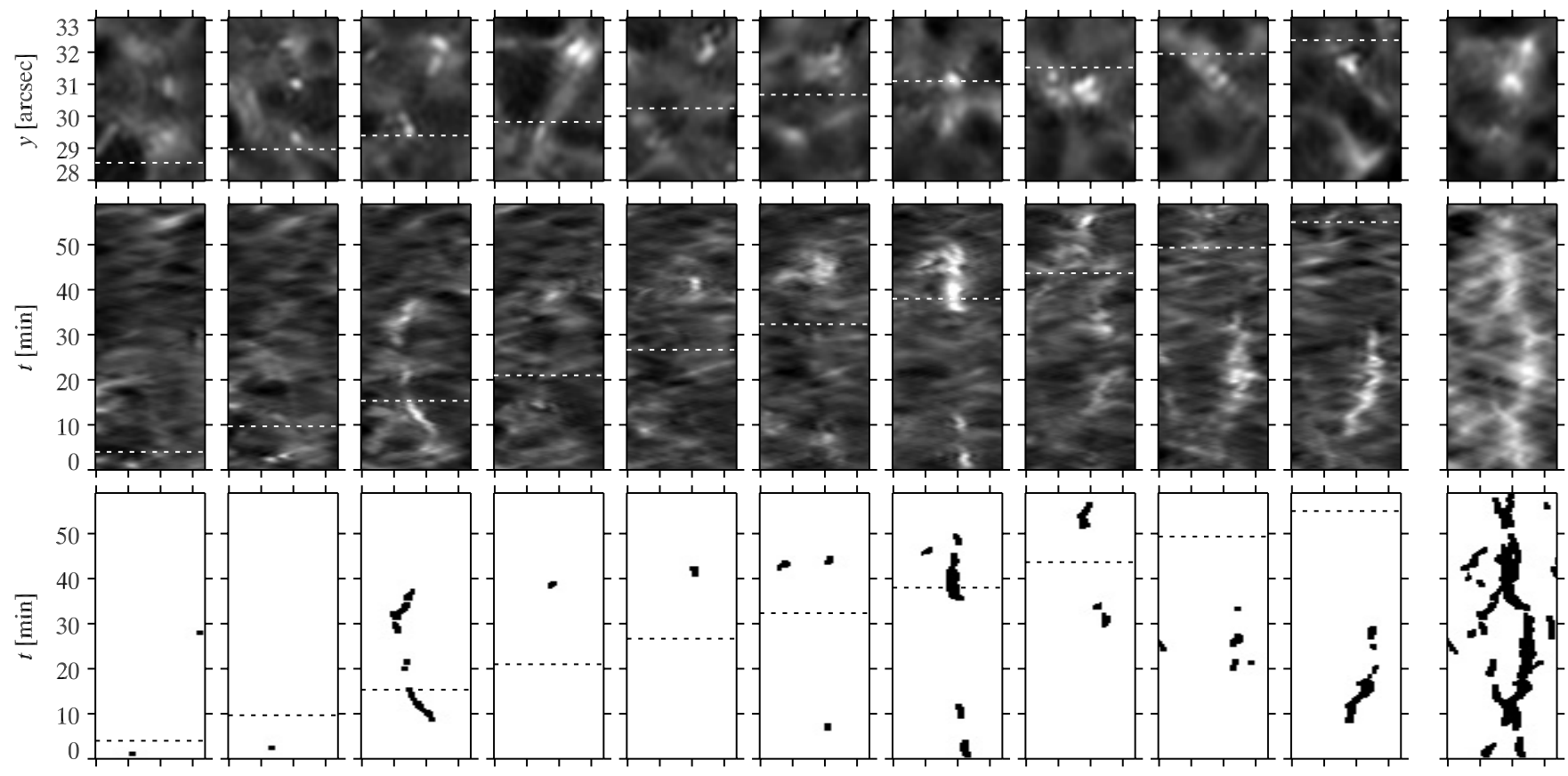

$\begin{array}{llllllllllllllllllllll}4 & 5 & 6 & 7 & 4 & 5 & 6 & 7 & 4 & 5 & 6 & 7 & 4 & 5 & 6 & 7 & 4 & 5 & 6 & 7 & 4 & 5\end{array}$

$x$ [arcsec]

Fig. 2. Example of a magnetic patch, visualized by partial Ca II $\mathrm{H}$ images ( $x-y$ cutouts, top row), the associated Ca II $\mathrm{H} x$ - $t$ slices (middle row), and the corresponding $x-t$ slices from the IBP map sequence (bottom row). The images shown here are a selection of the data at intervals of $\Delta t=340 \mathrm{~s}$ starting at $t=240 \mathrm{~s}$ and $\Delta y=0.426^{\prime \prime}$ starting at $y=0.568^{\prime \prime}$, respectively. The rightmost panels are averages over all data collected in the duration of the sequence (top panel) or in the $y$ interval shown here (lower panels). The $y$ location of each $x-t$ slice is shown by a dashed line in the associated $x-y$ panel, while the time of the latter is shown by a dashed line in the corresponding $x-t$ slice. 
over the plotted $t$ and $y$ ranges. We discuss two representative cases. (i) The first one is the bright IBP near $y \approx 29.5 \operatorname{arcsec}$ in the third $x-y$ panel $(t \approx 15 \mathrm{~min})$, which is sampled by the corresponding $x-t$ slice. The latter indicates an IBP lifetime of about $7 \mathrm{~min}$, during which it migrated leftward at about $0.15 \operatorname{arcsec~min}^{-1}$, or $1.8 \mathrm{~km} \mathrm{~s}^{-1}$. It seems to be fairly isolated, except that some intermittent brightness appears later at $x=5-6 \operatorname{arcsec}$ in the same $x-t$ slice. The next three $x-t$ slices show a bright structure near $t \approx 40$ min that migrates toward larger $x$. (ii) The second example is the bright grain near $y \approx 31$ arcsec in the 7 th $x-y$ panel for $t \approx 38$ min. Its $x-t$ slice indicates that it appeared abruptly at this time and then lived for fifteen minutes, but the surrounding slices show enhanced brightness nearby in $y$ before and after as well. True cube slicing confirms that the first example is indeed a continuously present magnetic structure which merges at $t \approx 45 \mathrm{~min}$ with the second example.

The rightmost panels of Fig. 2 encompass the third dimension through integration over the full sequence duration (top panel) or over the $y$ range shown (middle panel). The nearly continuous brightness in the interval $x=5-7$ arcsec suggests that many IBPs in this area belong to a common magnetic patch throughout the $\mathrm{Ca}$ II $\mathrm{H}$ sequence duration. Figure 4 confirms that this is indeed the case with the friends-of-friends patch definition described below. Note that the IBPs of cases (i) and (ii) apparently intersect in this panel at $t \approx 12 \mathrm{~min}$, whereas they are in fact disjunct in $y$ (as is shown in detail by the $x-y$ panels in Fig. 2 and by the $x$-integrated $y-t$ slice in Fig. 4).

We have developed a detection algorithm to locate magnetic patches made up by IBPs in order to quantify our visual impressions from cube slicing. It includes handling of splits and mergers, such as the ones discussed above. First, a mask to block off the network was constructed from the $\mathrm{Ca}$ II $\mathrm{H}$ sequence by temporal averaging of the entire one-hour Ca II $\mathrm{H}$ sequence, followed by 50-pixel boxcar smoothing and thresholding at the mean value, passing only lower values. The resulting mask is shown in Fig. 3. The selection is conservative in that medium-bright areas around bright network (the "intermediate" pixel class of Krijger et al. 2001 and Rutten et al. 2004a) are also rejected.

We next employed a multi-step procedure to locate IBPs in the masked-off sequences. Each image was first convolved with a suitable kernel to increase the contrast of small round features. The kernel has the form

$K_{D}(r)=\cos ^{2}\left(\frac{2 \pi r}{D}\right)-\kappa$

where $\kappa$ is a chosen such that the spatial average $\left\langle K_{D}(r)\right\rangle=0$. For the G-band sequence we used a kernel with a 5-pixel diameter $(D=4)$. We chose a 7-pixel kernel $(D=6)$ for the Ca II H images, in which IBPs are larger. We subsequently produced binary maps of IBP-candidates by thresholding the convolved images at a suitable level. This threshold level must be low, because IBPs occasionally become very weak between periods of enhanced brightness. The resulting maps therefore are quite noisy and require further processing.

The binary map sequences were improved further through spatial and temporal erosion-dilation processing. A spatial

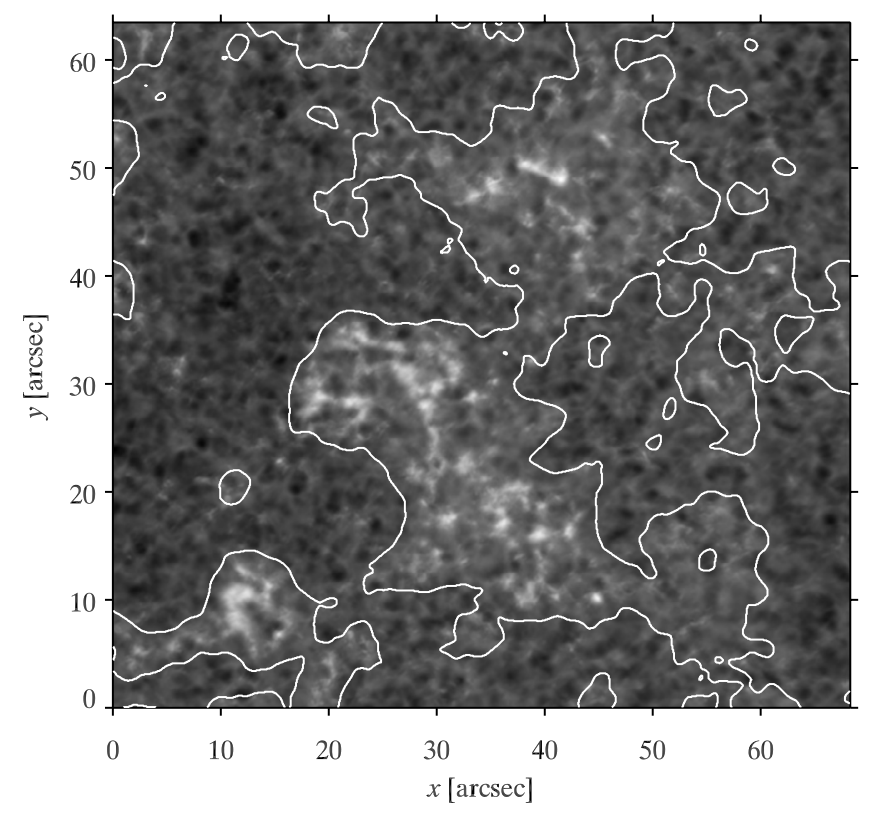

Fig. 3. The temporal average of the $\mathrm{Ca}$ II $\mathrm{H}$ image sequence over its one-hour duration with the internetwork mask contours overlaid in white. The Ca II H image intensity was scaled logarithmically in order to show contrast in the internetwork. The internetwork mask is computed by taking a 50-pixel boxcar average and thresholding at the mean value.

erosion operation tests the local nature of a candidate IBP in a binary map by discarding those pixels whose surroundings do not match a given kernel. A dilation operation does the opposite by adding pixels around the candidate IBP pixels. For the Ca II H maps, we chose a $3 \times 3$-pixel kernel for the erosion as well as the dilation operation, so that only candidate IBPs of at least this size pass the test. This spatial processing was omitted for the G-band maps, because G-band IBPs are often smaller than this kernel. However, temporal erosion-dilation processing was done on both binary-map sequences using a kernel of three time steps $(60 \mathrm{~s})$ to remove short-lived features.

Although the erosion-dilation processing removes much noise, there remain structures in the binary IBP maps that are not IBPs. Reversed granulation (cf. Rutten et al. 2004a) in particular produces arc-shaped structures in the binary Ca II $\mathrm{H}$ maps that the above processing fails to remove. Many of these are short-lived. We therefore discarded all structures with lifetimes less than $80 \mathrm{~s}$ (4 images).

In order to retain only features with a small spatial extent, we keep only those features whose average maximum instantaneous size in $x$ or $y$ expressed in units of $0.071^{\prime \prime}$ pixels is smaller than the feature lifetime measured in units of 20-s sampling intervals. Finally, we inspected all candidate IBPs visually, either accepting or discarding them. We believe that this last, laborious step removes most, if not all of the misidentifications from our sample.

The third row in Fig. 2 shows the corresponding $x-t$ slices through the resulting binary Ca II H IBP map sequence. Much of the brightness pattern in the rightmost slice in the middle row survives the IBP selection. The IBPs of case (i) and (ii) 

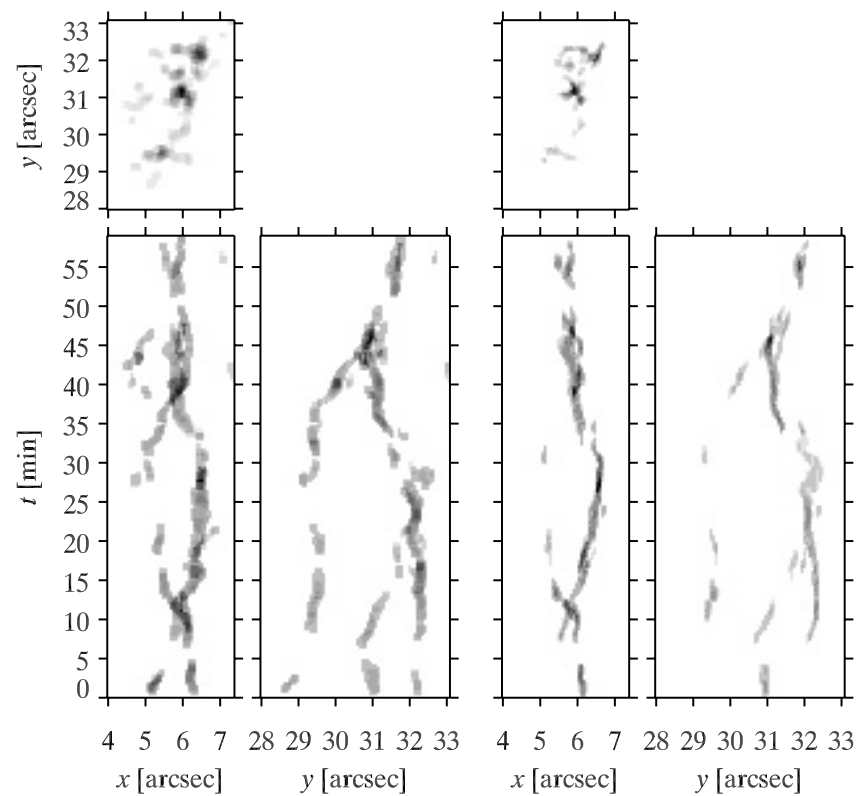

Fig. 4. Integrated $x-y$ cutout, and $x-t$ and $y-t$ slices corresponding to those in Fig. 2 of the binary IBP map sequences for the Ca II H (left) and $\mathrm{G}$ band (right), showing only those IBPs that group into the central patch. Careful comparison with the rightmost panel in the bottom row of Fig. 2 shows that several IBPs present there are part of another patch, such as at the leftmost edge of the cutout around $t \approx 25 \mathrm{~min}$. Each panel displays the integrated IBP map sequence over the third coordinate, i.e., the $x-y$ map sequence cutouts integrate over time, the $x-t$ slices over $y$, and the $y-t$ slices over $x$.

are, of course, properly detected by our algorithm. The apparent intersection at $t \approx 12 \mathrm{~min}$ also remains. However, some IBPs identified by our algorithm, for example those around $x \approx 5 \operatorname{arcsec}$ and $t \approx 20 \mathrm{~min}$, are not visible in the $y$-averaged Ca II H brightness.

The next step is to group the IBPs into magnetic patches. To this end, we apply a friends-of-friends algorithm. Two IBPs are considered friends if their minimum separation is less than $0.71^{\prime \prime}$ (10 pixels), with disregard of temporal separation. A patch then consists of a group of befriended IBPs that have no friends outside the group. It may contain IBPs that are not direct friends if a string of IBPs form a path connecting them. Each IBP is associated with a single patch, but a patch often contains multiple IBPs.

The left-hand part of Fig. 4 shows the result of the patch analysis for the $\mathrm{Ca}$ II $\mathrm{H}$ sequence cutout shown in Fig. 2. The three panels display the corresponding $x-y$ cutout, as well as the associated $x-t$ and $y-t$ slices through the binary cube made up of the $24 \mathrm{Ca}$ II H IBPs that are members of this particular patch. Each panel is integrated over $x, y$, or $t$, as appropriate, so that a larger blackness signifies the presence of more IBP pixels in the cube along the third dimension. Some IBPs visible in Fig. 2 are missing, such as the one around $x \approx 4 \operatorname{arcsec}$ and $t \approx 25 \mathrm{~min}$, because they are not a member of this patch. The $y$-integrated $x-t$ slice in the bottom-left panel shows the intersection at $t \approx 12 \mathrm{~min}$ and the merger at $t \approx 45 \mathrm{~min}$ that were already visible in the rightmost panels of Fig. 2. Adding

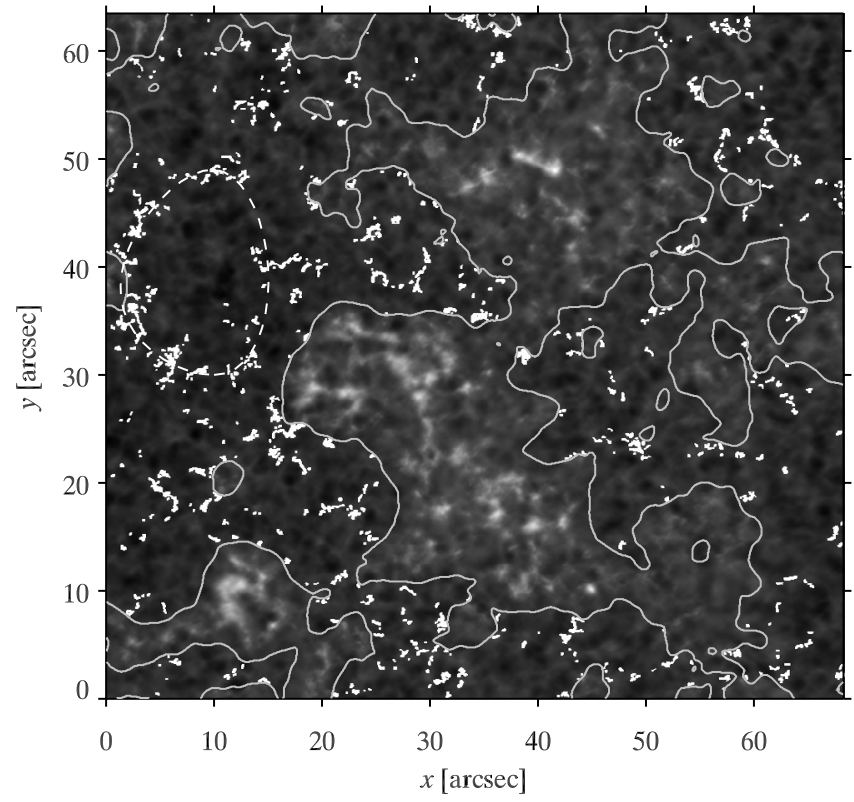

Fig. 5. The average $\mathrm{Ca} I \mathrm{H}$ image with IBPs identified in the $\mathrm{Ca}$ II $\mathrm{H}$ sequence overlaid in white. The IBPs appear to group in patches that outline edges of cell-like structures, such as around $(x, y)=\left(10^{\prime \prime}, 40^{\prime \prime}\right)$ (indicated by a dashed line).

the information in the $y-t$ slice makes it clear that there are in fact three trails, with mergers at $t \approx 20 \mathrm{~min}$ and $t \approx 45 \mathrm{~min}$. The apparent intersection in the $x-t$ slice at $t \approx 12 \mathrm{~min}$ is actually a disjunct crossing, but the merger at $t \approx 45 \mathrm{~min}$ is real. The latter connects the two legs of the patch through the friends-offriends labeling.

Figure 4 also adds the corresponding triplet for the $\mathrm{G}$ band map sequence (three right-hand panels). There is a strong correlation between the two diagnostics, but $\mathrm{Ca}$ II $\mathrm{H}$ indeed provides more IBPs. The right-hand trail is very similar in the two $y-t$ slices, but the left-hand trail mostly vanishes in the G-band slice.

\section{Results and discussion}

\subsection{Patch patterns}

The resulting IBP collection consists of 387 features in the G-band map sequence that are classified as IPBs, and 848 IBPs in the $\mathrm{Ca}$ II $\mathrm{H}$ map sequence. There are more in the latter because IBPs are more easily identified in Ca II H. We find 149 G-band patches, 76 of which contain multiple IBPs, and $217 \mathrm{Ca}$ II $\mathrm{H}$ patches, 125 of which contain multiple IBPs. A comparison shows that $85 \%$ of the G-band patches coincide spatially with $\mathrm{Ca}$ II $\mathrm{H}$ patches.

The locations of all Ca II H IBPs, irrespective of their time of appearance, are shown in Fig. 5. They show a striking pattern in which groups of IBPs appear to partially outline the edges of cell-like structures of mesogranular scale, e.g., around $(x, y)=$ $\left(10^{\prime \prime}, 40^{\prime \prime}\right)$, where a large, conspicuous cell is marked with a dashed curve, around (20", 15"), and around (27", $\left.42^{\prime \prime}\right)$. 


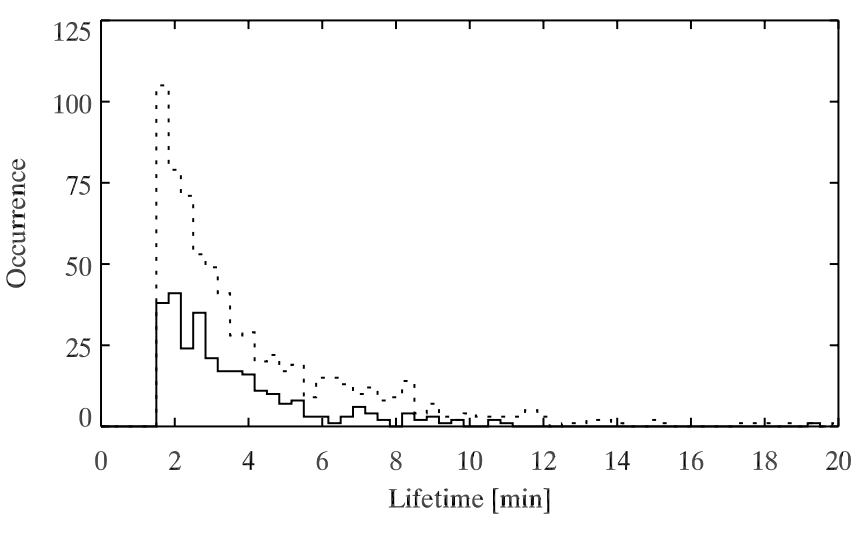

Fig. 6. Histograms of IBP lifetimes. Solid: G-band IBP lifetimes. Dotted: Ca II H IBP lifetimes. IBPs with lifetimes shorter than 4 time steps ( $80 \mathrm{~s}$ ) are rejected by our processing.

Similar patterns have been reported before in studies of internetwork magnetograms (Domínguez Cerdeña et al. 2003; Sánchez Almeida 2003; Domínguez Cerdeña 2003). In particular, Domínguez Cerdeña (2003) analyzed a 17-min sequence of Fe I magnetograms and found that the stronger internetwork magnetic elements show a pattern that coincides with mesogranular upwellings. One may expect such a pattern to be set by underlying granular motions. Roudier \& Muller (2004) advected corks by measured granular flows and found that the corks concentrate at the boundaries of "trees of fragmenting granules". These were previously connected to mesogranules by Roudier et al. (2003), who found that such trees may have lifetimes of many hours.

\subsection{IBP lifetimes and number density}

Figure 6 shows a histogram of the durations over which our algorithm tracks the IBPs that are completely within the temporal and spatial boundaries, and that do not split or merge. We find an average IBP lifetime of $3.5 \mathrm{~min}$ in the $\mathrm{G}$ band and $4.3 \mathrm{~min}$ in Ca II H. Both the G-band and Ca II H IBP lifetime distributions show a long tail towards long lifetimes, with maxima of $19.3 \mathrm{~min}$ and $25.3 \mathrm{~min}$, respectively.

Berger et al. (1998b) find longer lifetimes of $9.3 \mathrm{~min}$ for G-band network bright points. Possibly, their data and analysis permit better tracking of NBPs. Their method of NBP detection in G-band images by subtraction of a suitably scaled continuum image yields a much improved contrast between granulation and NBPs. However, there is no equivalent method for $\mathrm{Ca}$ II $\mathrm{H}$ images. In addition, internetwork bright points generally have substantially lower contrast than network bright points, and are therefore much harder to identify and track. By reducing the threshold value in our data reduction, IBPs mightbe followed through periods of lower intensity, which somewhat increases the mean lifetime at the price of many more misidentifications.

Bright point lifetimes in internetwork regions may be shorter than in network regions. Where it is strong, the network disturbs the convection by fragmenting granules into "abnormal granulation", so that flux tubes in network areas have a relatively quiet life compared to those in internetwork areas, where full-size granules continuously crash into them. Their rapid, erratic movements make internetwork IBPs harder to track, and are furthermore likely to disturb the processes that make them bright. Since IBPs cluster in patches, we conclude that, even though the IBP may momentarily become invisible, the magnetic field element remains and may become bright again at some later time. This agrees well with the conclusion of Berger \& Title (2001) that magnetism is a necessary but not a sufficient condition for the formation of a network bright point. We find an average IBP number density of $0.02 \mathrm{Mm}^{-2}$ in the G-band images and $0.05 \mathrm{Mm}^{-2}$ in the $\mathrm{Ca}$ II $\mathrm{H}$ images. This is an order of magnitude less than the result of Sánchez Almeida et al. (2004), who find a number density of $0.3 \mathrm{Mm}^{-2}$ in their best G-band image. They manually located bright points in data with higher resolution than used in this analysis, which possibly allowed them to identify fainter features than our algorithm. Also, their $23^{\prime \prime} \times 35^{\prime \prime}$ image contains a small network patch. We exclude all network and a fair area around it, where the bright point number density is increased (cf. Fig. 2 of Sánchez Almeida et al. 2004).

\subsection{Statistical patch lifetime estimation}

Visual inspection of the patches identified by our algorithm shows that only a few patches begin or end within the duration of the sequence, indicating that their typical lifetime substantially exceeds one hour. We therefore apply statistical arguments to obtain an estimate for the lifetime.

Assume that we observe a patch with lifetime $\tau$ in a sequence lasting $t_{\mathrm{obs}}$. The patch is visible if the sequence starts between $t_{\mathrm{obs}}$ before the patch emerges and the time that the patch disappears, i.e., in order to see the patch, the sequence must start in a time window of $\tau+t_{\mathrm{obs}}$. We distinguish three cases.

(i) A patch persisting throughout the entire sequence. If the patch has a lifetime $\tau \geq t_{\mathrm{obs}}$, it is only visible for the entire duration of the sequence if it emerges up to $\tau-t_{\text {obs }}$ before the start of the sequence. Therefore, the probability of finding such a patch is given by

$p_{1}(\tau)= \begin{cases}0 & \text { if } \tau \leq t_{\mathrm{obs}} \\ \frac{\tau-t_{\mathrm{obs}}}{\tau+t_{\mathrm{obs}}} & \text { if } \tau \geq t_{\mathrm{obs}}\end{cases}$

(ii) A patch that emerges as well as disappears within the duration of the sequence. A patch with lifetime $\tau \leq t_{\mathrm{obs}}$ emerges as well as disappears within the duration of the sequence if it emerges up to $t_{\mathrm{obs}}-\tau$ after the sequence starts. The probability thus is

$p_{2}(\tau)= \begin{cases}\frac{t_{\mathrm{obs}}-\tau}{\tau+t_{\mathrm{obs}}} & \text { if } \tau \leq t_{\mathrm{obs}} \\ 0 & \text { if } \tau \geq t_{\mathrm{obs}}\end{cases}$

(iii) A patch that either emerges or disappears within the duration of the sequence. In case $\tau \geq t_{\mathrm{obs}}$, a patch is seen to emerge, but not to disappear, if the observation is started up to $t_{\mathrm{obs}}$ before the patch emerges. Similarly, it is seen to disappear, but not to appear, if the observation is started up to $t_{\mathrm{obs}}$ 
before the patch disappears. In the case that $\tau \leq t_{\mathrm{obs}}$, the patch is seen to emerge, but not to disappear, if the patch emerges up to $\tau$ before the end of the observation, and is seen to disappear, but not to emerge, if the patch disappears up to $\tau$ after the start of the observation. The probability $p_{3}$ of seeing a patch with lifetime $\tau$ either emerge or disappear in a sequence of duration $t_{\mathrm{obs}}$ thus is

$p_{3}(\tau)= \begin{cases}\frac{2 \tau}{\tau+t_{\mathrm{obs}}} & \text { if } \tau \leq t_{\mathrm{obs}}, \\ \frac{2 t_{\mathrm{obs}}}{\tau+t_{\mathrm{obs}}} & \text { if } \tau \geq t_{\mathrm{obs}} .\end{cases}$

We obviously have $p_{1}(\tau)+p_{2}(\tau)+p_{3}(\tau)=1$.

The expected numbers of patches $N_{i}^{\prime}$ with $i=1,2,3$ can be computed if we assume a lifetime distribution. We adopt a reasonable choice of an exponential distribution,

$D_{\lambda}(\tau)=\lambda \mathrm{e}^{-\lambda \tau}$

This distribution has one adjustable parameter, $\lambda$, that is a measure of the decay time scale of the distribution. The expected numbers of patches then follow from integrals of the form

$N_{i}^{\prime}=N \int_{0}^{\infty} p_{i}(\tau) D_{\lambda}(\tau) \mathrm{d} \tau$,

where $N$ is the total number of patches observed.

To obtain a fit for the parameter $\lambda$, we need to count the number of patches of each type in our sequence. In nearly all cases a patch can be traced much longer by eye than that it is identified by the algorithm described in Sect. 2. We therefore visually inspected the original data and identified those patches that remain visible during the whole sequence $\left(N_{1}=\right.$ 124), those that both emerge and disappear during the sequence $\left(N_{2}=11\right)$, and those that either emerge or disappear $\left(N_{3}=68\right)$. We discarded the 14 patches that enter or exit the field of view during the sequence. We find an excellent fit for $\lambda=1.9 \times 10^{-3} \mathrm{~min}^{-1}$, yielding $N_{1}^{\prime}=123.7, N_{2}^{\prime}=8.6$, and $N_{3}^{\prime}=70.8$. The excellent fit provides confidence in the validity of the distribution $D_{\lambda}(\tau)$. The average patch lifetime is then given by

$\langle\tau\rangle=\int_{0}^{\infty} \tau D_{\lambda}(\tau) \mathrm{d} \tau=\frac{1}{\lambda} \approx 530 \pm 50 \mathrm{~min}$,

where the error was estimated by variation of $N_{i}$ within their error bounds set by Poisson statistics.

\section{Conclusion}

We have found numerous IBPs in photospheric and chromospheric quiet-sun internetwork cells. Our results show that Ca II $\mathrm{H}$ line-core filtergrams are well-suited for finding magnetic flux tubes in the quiet-sun internetwork. IBPs in our $\mathrm{Ca}$ II $\mathrm{H}$ images show a good correlation with G-band IBPs. We follow Sánchez Almeida et al. (2004) in attributing both IBPs in the G-band and $\mathrm{Ca}$ II $\mathrm{H}$ sequences to kiloGauss magnetic flux tubes.

The IBP density that we measure is significantly lower than the value of Sánchez Almeida et al. (2004), which is likely due to our lower resolution and more conservative identification method.

We find that magnetic IBPs cluster into patches that are not homogeneously distributed over the internetwork, but rather seem to outline cell-like structures similar to the magneticelement voids found by Domínguez Cerdeña et al. (2003) marking mesogranular upwellings. This apparent mesogranular distribution indicates that the magnetic elements which intermittently appear as IBPs in a patch have a sufficient long lifetime to assemble at mesogranular vertexes. The strongest may eventually make it as NBPs to the supergranular boundaries. Indeed, all IBP patches identified by our algorithm exist on time scales much larger than granular time scales. This result seems to exclude their attribution to a granular dynamo as described by, e.g., Cattaneo (1999).

Through statistical analysis, we estimate an average lifetime of about nine hours. This estimate depends on a distribution that admittedly cannot be verified from these observations. This would require observations over much longer duration $(>10 \mathrm{~h})$. The Solar-B mission in its sun-synchronous polar orbit will provide the high-resolution seeing-free longduration observations that these analyses require.

Acknowledgements. The DOT is operated by Utrecht University at the Spanish Observatorio del Roque de los Muchachos of the Instituto de Astrofísica de Canarias and is presently funded by Utrecht University, the Netherlands Organisation for Scientific Research NWO, the Netherlands Graduate School for Astronomy NOVA, and SOZOU. The DOT efforts are part of the European Solar Magnetism Network.

\section{References}

Beckers, J. M., \& Schröter, E. H. 1968, Sol. Phys., 4, 142

Berger, T. E., \& Title, A. M. 1996, ApJ, 463, 365

Berger, T. E., \& Title, A. M. 2001, ApJ, 553, 449

Berger, T. E., Schrijver, C. J., Shine, R. A., et al. 1995, ApJ, 454, 531

Berger, T. E., Löfdahl, M. G., Shine, R. A., \& Title, A. M. 1998a, ApJ, 506, 439

Berger, T. E., Löfdahl, M. G., Shine, R. A., \& Title, A. M. 1998b, ApJ, 495, 973

Berger, T. E., Rouppe van der Voort, L. H. M., Löfdahl, M. G., et al. 2004, A\&A, 428, 613

Brandt, P. N., Rutten, R. J., Shine, R. A., \& Trujillo Bueno, J. 1992, in Cool Stars, Stellar Systems, and the Sun, ASP Conf. Ser., 26, 161

Brandt, P. N., Rutten, R. J., Shine, R. A., \& Trujillo Bueno, J. 1994, in Solar Surface Magnetism, 251

Carlsson, M., \& Stein, R. F. 1997, ApJ, 481, 500

Carlsson, M., Stein, R. F., Nordlund, Å., \& Scharmer, G. B. 2004, ApJ, 610, L137

Cattaneo, F. 1999, ApJ, 515, L39

Cattaneo, F., Emonet, T., \& Weiss, N. 2003, ApJ, 588, 1183

Chapman, G. A., \& Sheeley, N. R. 1968, Sol. Phys., 5, 442

Domínguez Cerdeña, I. 2003, A\&A, 412, L65

Domínguez Cerdeña, I., Kneer, F., \& Sánchez Almeida, J. 2003, ApJ, 582, L55

Dunn, R. B., \& Zirker, J. B. 1973, Sol. Phys., 33, 281

Emonet, T., \& Cattaneo, F. 2001, ApJ, 560, L197

Frazier, E. N., \& Stenflo, J. O. 1972, Sol. Phys., 27, 330

Grossmann-Doerth, U., Knölker, M., Schüssler, M., \& Solanki, S. K. 1994, A\&A, 285, 648 
Grossmann-Doerth, U., Schüssler, M., \& Steiner, O. 1998, A\&A, 337, 928

Heyvaerts, J., \& Priest, E. R. 1983, A\&A, 117, 220

Howard, R., \& Stenflo, J. O. 1972, Sol. Phys., 22, 402

Keller, C. U., Steiner, O., Stenflo, J. O., \& Solanki, S. K. 1990, A\&A, 233, 583

Keller, C. U., Schüssler, M., Vögler, A., \& Zakharov, V. 2004, ApJ, 607, L59

Knölker, M., \& Schüssler, M. 1988, A\&A, 202, 275

Krijger, J. M., Rutten, R. J., Lites, B. W., et al. 2001, A\&A, 379, 1052

Lites, B. W., Rutten, R. J., \& Berger, T. E. 1999, ApJ, 517, 1013

Lites, B. W., \& Socas-Navarro, H. 2004, ApJ, 613, 600

Livingston, W., \& Harvey, J. 1969, Sol. Phys., 10, 294

Löfdahl, M. G., Berger, T. E., Shine, R. A., \& Title, A. M. 1998, ApJ, 495, 965

Manso Sainz, R., Landi Degl'Innocenti, E., \& Trujillo Bueno, J. 2004, ApJ, 614, L89

Mehltretter, J. P. 1974, Sol. Phys., 38, 43

Muller, R. 1977, Sol. Phys., 52, 249

Muller, R. 1983, Sol. Phys., 85, 113

Muller, R., \& Roudier, T. 1984, Sol. Phys., 94, 33

Nindos, A., \& Zirin, H. 1998, Sol. Phys., 179, 253

Parker, E. N. 1988, ApJ, 330, 474

Roudier, T., Lignières, F., Rieutord, M., Brandt, P. N., \& Malherbe, J. M. 2003, A\&A, 409, 299

Roudier, T., \& Muller, R. 2004, A\&A, 419, 757

Rouppe van der Voort, L. H. M., Hansteen, V. H., Carlsson, M., et al. 2005, A\&A, 435, 327

Rutten, R. J., \& Uitenbroek, H. 1991, Sol. Phys., 134, 15

Rutten, R. J., Kiselman, D., Rouppe van der Voort, L., \& Plez, B. 2001, in Advanced Solar Polarimetry - Theory, Observation, and Instrumentation, ASP Conf. Ser., 236, 445
Rutten, R. J., de Wijn, A. G., \& Sütterlin, P. 2004a, A\&A, 416, 333

Rutten, R. J., Hammerschlag, R. H., Bettonvil, F. C. M., Sütterlin, P., $\&$ de Wijn, A. G. 2004b, A\&A, 413, 1183

Sánchez Almeida, J. 2003, A\&A, 411, 615

Sánchez Almeida, J., Domínguez Cerdeña, I., \& Kneer, F. 2003, ApJ, 597, L177

Sánchez Almeida, J., Márquez, I., Bonet, J. A., Domínguez Cerdeña, I., \& Muller, R. 2004, ApJ, 609, L91

Schrijver, C. J., \& Title, A. M. 2003, ApJ, 597, L165

Sivaraman, K. R., \& Livingston, W. C. 1982, Sol. Phys., 80, 227

Sivaraman, K. R., Gupta, S. S., Livingston, W. C., et al. 2000, A\&A, 363,279

Socas-Navarro, H., \& Lites, B. W. 2004, ApJ, 616, 587

Socas-Navarro, H., Martínez Pillet, V., \& Lites, B. W. 2004, ApJ, 611, 1139

Solanki, S. K., \& Brigljevic, V. 1992, A\&A, 262, L29

Spruit, H. C. 1976, Sol. Phys., 50, 269

Spruit, H. C. 1977, Ph.D. Thesis

Spruit, H. C., \& Zwaan, C. 1981, Sol. Phys., 70, 207

Steiner, O. 2005, A\&A, 430, 691

Steiner, O., Grossmann-Doerth, U., Knölker, M., \& Schüssler, M. 1998, ApJ, 495, 468

Stenflo, J. O. 1973, Sol. Phys., 32, 41

Trujillo Bueno, J., Shchukina, N., \& Asensio Ramos, A. 2004, Nature, 430, 326

van Ballegooijen, A. A., Nisenson, P., Noyes, R. W., et al. 1998, ApJ, 509,435

von Uexkuell, M., \& Kneer, F. 1995, A\&A, 294, 252

Wiehr, E., Bovelet, B., \& Hirzberger, J. 2004, A\&A, 422, L63

Wilson, P. R. 1981, Sol. Phys., 69, 9

Worden, J., Harvey, J., \& Shine, R. A. 1999, ApJ, 523, 450

Zwaan, C. 1967, Sol. Phys., 1, 478 